\title{
Preparation, characterization and relative bioavailability of oral elemene o/w microemulsion
}

This article was published in the following Dove Press journal:

International Journal of Nanomedicine

5 August 2010

Number of times this article has been viewed

\section{Zhaowu Zeng' \\ Guanglin Zhou' \\ Xiaoli Wang ${ }^{2}$ \\ Eric Zhijian Huang' \\ Xiaori Zhan' \\ Jun Liu' \\ Shuling Wang' \\ Anming Wang' \\ Haifeng $\mathrm{Li}^{\prime}$ \\ Xiaolin Pei ${ }^{1}$ \\ Tian Xie'}

'Research Center for Biomedicine and Health, Hangzhou Normal

University, Hangzhou, Zhejiang,

China; ${ }^{2}$ Yichun University of Jiangxi

Province, Yichun, Jiangxi, China
Correspondence: Tian Xie

Research Center for Biomedicine and Health, Hangzhou Normal University, 222 Wenyi Road, Hangzhou, Zhejiang 310012 , PR China

Tel $+8657 \mid 28861252$

Email artgreenking@I26.com
Abstract: The objective was to develop an elemene oil/water $(\mathrm{o} / \mathrm{w})$ microemulsion and evaluate its characteristics and oral relative bioavailability in rats. Elemene was used as the oil phase and drug, polysorbate 80 as a surfactant along with ethanol, propylene glycol, and glycerol as the cosurfactants. The microemulsion was prepared by mixing method, or ultrasonication method in an ultrasonic bath. Its three-dimensional response surface diagram was drawn by Mathcad software. The microemulsion was characterized by visual observation, cross-polarized microscopy, size, zeta potential, acidity, viscosity, and surface tension measurement. The drug content and entrapment efficiency were determined by ultra fast liquid chromatography (UFLC) and liquid surface method. Blood was drawn from rats at different time points after oral administration of an elemene microemulsion or a commercial elemene emulsion for measurement of the drug in plasma by UFLC to establish the pharmacokinetic parameters and relative bioavailability. The elemene microemulsion as a clarified and isotropic system containing $1 \%$ elemene $(\mathrm{w} / \mathrm{v}), 5 \%$ ethanol (v/v), 15\% propylene glycol (v/v), 15\% glycerol (v/v), and $5 \%$ polysorbate $80(\mathrm{w} / \mathrm{v})$, was characterized as $(57.7 \pm 2.8) \mathrm{nm}$ in size, $0.485 \pm 0.032$ in polydispersity index, $(3.2 \pm 0.4) \mathrm{mv}$ in zeta potential, $(5.19 \pm 0.08) \mathrm{in} \mathrm{pH}, 6 \mathrm{mpa} \cdot \mathrm{s}$ in viscosity, $(31.8 \pm 0.3) \mathrm{mN} \cdot \mathrm{m}^{-1}$ in surface tension, $(8.273 \pm 0.018) \mathrm{mg} \cdot \mathrm{mL}^{-1}$ in content of $\beta$-elemene, and $(99.81 \pm 0.24) \%$ in average entrapment efficiency. The area under the concentration-time curves from $0 \mathrm{~h}$ to $24 \mathrm{~h}$ $\left(\mathrm{AUC}_{0 \rightarrow 24 \mathrm{~h}}\right)$ of the elemene microemulsion and commercial elemene emulsion were integrated to be $3.092 \mathrm{mg} \cdot \mathrm{h} \cdot \mathrm{L}^{-1}$ and $1.896 \mathrm{mg} \cdot \mathrm{h} \cdot \mathrm{L}^{-1}$ respectively, yielding a relative bioavailability of $163.1 \%$. The present study demonstrates the elemene microemulsion as a new formulation with ease of preparation, high entrapment efficiency, excellent clarity, good stability, and improved bioavailability.

Keywords: elemene, microemulsion, relative bioavailability

\section{Introduction}

Elemene is a mixture of sesquiterpene compounds extracted from ginger plants curcuma, with outstanding advantages of a broad anti-tumor spectrum, curative effect, and less adverse reaction. ${ }^{1}$ Recently elemene emulsion has been used widely in clinical treatment for malignant effusions, lung cancer, gastrointestinal cancer, brain cancer, gynecological cancer, breast cancer, skin cancer, and other tumors. ${ }^{2,3}$ Among its three major components of $\beta, \gamma, \delta$ isomers, $\beta$-elemene is the main active ingredient ${ }^{2}$ and thus used commonly for content determination of elemene. Elemene is a volatile oil with poor oral absorption and low bioavailability, ${ }^{4}$ limiting its clinical applications.

Microemulsion is a transparent or translucent preparation with low viscosity, good isotropic and thermodynamic stability upon spontaneous formation in appropriate 
proportion of an oil phase and a water phase, along with surfactants and cosurfactants. As a drug carrier system for oral, transdermal and ophthalmic administrations, it has certain advantages: (1) ease of preparation, filtration and preservation; (2) simultaneous solubilization of drugs of different levels of lipophilicity; (3) good drug dispersion, absorption, and bioavailability; (4) extended release time of water-soluble drugs; (5) low viscosity and no injection pain. ${ }^{5,6} \mathrm{O} / \mathrm{W}$ microemulsion drug delivery system has been normally used via oral and parenteral administration. ${ }^{7}$ However, the surfactants of oral microemulsion may stimulate gastrointestinal mucosa and cause changes in mucosal permeability leading to systemic chronic toxicity in long-term use. The cosurfactants may also cause some irritation and pharmacological activity that could limit clinical use of the oral microemulsion. ${ }^{8}$ So, we aimed to develop an oral elemene microemulsion formulation with excellent clarity, high entrapment efficiency, good patient compliance, and improved bioavailability while meeting the demand of facile industrial production. In this paper we describe the preparation, characteristics, and relative bioavailability of oral elemene o/w microemulsion.

\section{Materials and methods}

\section{Instruments and materials}

XR-UFLC equipped with a $\mathrm{C}_{18}$ column $(2.2 \mu \mathrm{m}, 75 \mathrm{~mm} \times$ $3.0 \mathrm{~mm}$ ) was purchased from Shimadzu Corporation (Tokyo, Japan). KQ5200B Ultrasonic cleaner (200 watt) was from Kunshan Ultrasound Instruments Co, Ltd (Kunshan, Shanghai, China). Sigma 3k30 Centrifuge was from Sartorius Company (Göttingen, German). Nikon E600 POL cross-polarized light microscopy was from Nikon Co. (Tokyo, Japan). Zetasizer 3000HS nano size and potential analyzer was from Malvern Instruments Ltd. (Malvern, Worcestershire, England). DELTA320 pH meter was from Mettler-Toledo Co. (Zurich, Switzerland). NDJ-5S digital viscometer and BZY-A automatic surface tension meter were from Shanghai Ruifang Instrument Co, Ltd (Shanghai, China). Disposable needle filters $(0.22 \mu \mathrm{m})$ were from Shanghai Xingya Purification Material Factory (Shanghai, China). $\beta$-elemene standard was from National Institute for the Control of Pharmaceutical and Biological Products (No. 100268-200401, Beijing, China). Elemene crude drug and oral emulsions $(0.2 \mathrm{~g} / 20 \mathrm{~mL}$, No. 0904231) were from Dalian Holley King Kong Pharmaceutical Co. Ltd (Dalian, Liaoning, China). Acetonitrile was from Merck \& Co. (Darmstadt, Germany). Sprague Dawley rats were from Zhejiang Academy of Medical Sciences (Certificate of Conformity: SCXK(Zhe) 2008-0033, Hangzhou,
Zhejiang, China). Experimental animals were treated according to Guideline of Laboratory Animal Care from Chinese Ministry of Science and Technology in $2006 .{ }^{9}$

\section{Three-dimensional response surface diagram of elemene microemulsion}

Elemene was used as the oil phase and drug, polysorbate 80 as the surfactant along with ethanol, propylene glycol as the cosurfactants, and glycerol as the tackifier and absorption enhancer. The amount of elemene and glycerol were fixed at $0.4 \mathrm{~g}$ and $6 \mathrm{~mL}$ respectively. Various volumes of ethanol $(0,2,4,6,8 \mathrm{~mL})$ and propylene glycol $(0,2$, $4,6,8 \mathrm{~mL}$ ) at a total of 25 combinations were taken to prepare elemene microemulsions. At $25^{\circ} \mathrm{C}$, a mixture of elemene, ethanol, propylene glycol, and glycerol was added into $25 \mathrm{~mL}$ of water, followed by dropwise addition of polysorbate 80 and mixing or ultrasonication until the formation of a clarified system. The final volume was adjusted to $50 \mathrm{~mL}$. In this process we found that these elemene microemulsions were easily formed by mixing method, but more easily by ultrasonication in an ultrasonic bath for greater clarity. So, we selected the ultrasonication in an ultrasonic bath to prepare these elemene microemulsions. The minimal amounts of polysorbate 80 required to maintain a clarified microemulsion in all 25 cases of ethanol-propylene glycol mixture was noted and used to construct the three-dimensional response surface diagram of elemene microemulsion by Mathcad 2001 software (Figure 1). The diagram shows that the minimal amount of polysorbate 80 for forming a microemulsion is decreased with increasing amounts of ethanol and propylene glycol. We were concerned with the safety issue of elemene microemulsion for the major side effects of oral polysorbate 80 over ethanol and propylene glycol and thus selected an elemene microemulsion with the lower dose of polysorbate 80 for further study.

\section{Preparation and characterization of elemene microemulsion}

According to the three-dimensional response surface diagram of elemene microemulsion, an optimal elemene microemulsion with minimal amount of polysorbate 80 was prepared. First, a mixture containing $1 \mathrm{~g}$ of elemene, $15 \mathrm{~mL}$ of glycerol, $5 \mathrm{~mL}$ of ethanol, $15 \mathrm{~mL}$ of propylene glycol, and $5 \mathrm{~g}$ of polysorbate 80 was added into $60 \mathrm{~mL}$ of water, followed by $1 \mathrm{~h}$ of ultrasonication in an ultrasonic bath and a volume adjustment to $100 \mathrm{~mL}$ with water. The isotropic nature of the microemulsion was examined using 


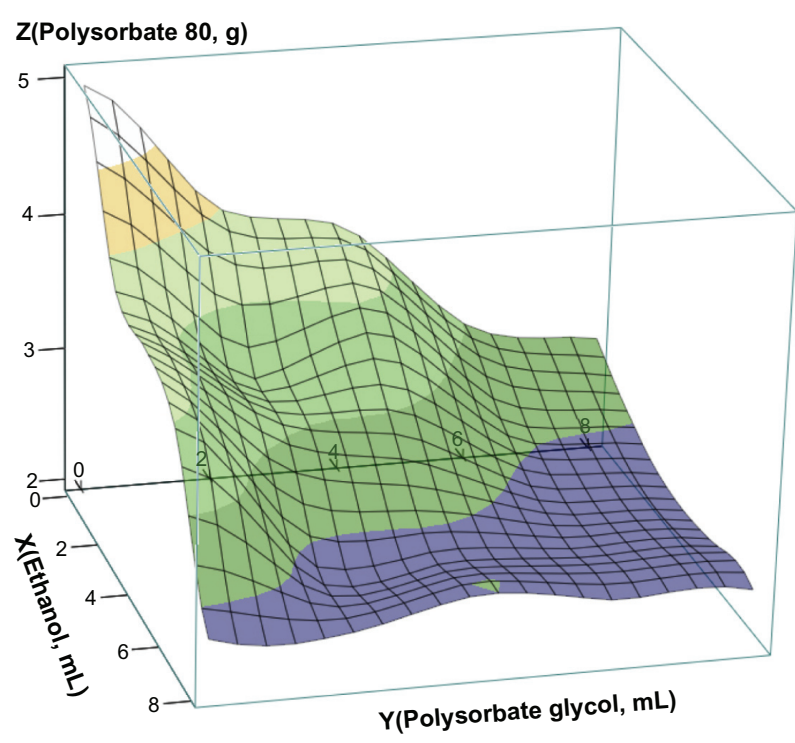

Figure I The three-dimensional response surface diagram of elemene microemulsion drawn by Mathcad software using the minimal amount of polysorbate 80 for forming a microemulsion.

cross-polarized light microscopy. Isotropic material, such as a microemulsion, does not interfere with the polarized light and should appear dark under cross-polarized microscope. ${ }^{10}$ The average particle size, polydispersity index, zeta potential, acidity, viscosity, and surface tension of this microemulsion were measured. Test temperature was $25^{\circ} \mathrm{C}$ in all cases.

\section{Stability of elemene microemulsion}

The elemene emulsion and microemulsion were centrifuged at $25^{\circ} \mathrm{C}$ and $3000 \times \mathrm{g}$ and $50000 \times \mathrm{g}$ respectively, for $30 \mathrm{~min}$ and followed by an examination for phase separation.

\section{UFLC analysis of elemene microemulsion}

According to the literature, ${ }^{11}$ the drug content was detected by UFLC. The chromatographic conditions were set as follows: $\mathrm{C}_{18}$ column $(2.2 \mu \mathrm{m}, 75 \mathrm{~mm} \times 3.0 \mathrm{~mm})$, acetonitrile/ water (80:20) as mobile phase, $40^{\circ} \mathrm{C}$ as column temperature, $210 \mathrm{~nm}$ as detection wavelength, $1.0 \mathrm{~mL} \cdot \mathrm{min}^{-1}$ as flow rate, and $5 \mu \mathrm{L}$ as injection volume. $\beta$-elemene standard was used as the external standard. Under such chromatographic conditions, the theoretical plate number of $\beta$-elemene was 9658 while the retention time of $\beta$-elemene was about $4.8 \mathrm{~min}$. No peak was found for a blank microemulsion without elemene at this retention time, indicating a specific detection of $\beta$-elemene. The standard curve of $\beta$-elemene concentration was linear in the range of $3.94-27.58 \mu \mathrm{g} \cdot \mathrm{mL}^{-1}$ with a regression equation of $\mathrm{Y}=2157.8318 * \mathrm{C}-311.4286$ $(\mathrm{r}=0.9996)$. The limit of detection and quantification was $0.6 \mathrm{ng}$ and $2 \mathrm{ng}$ respectively.

\section{Content and entrapment efficiency of $\beta$-elemene in elemene microemulsion}

To determine the drug content of elemene microemulsion, $0.1 \mathrm{~mL}$ of the elemene microemulsion was taken and diluted to $50 \mathrm{~mL}$ with $80 \%$ acetonitrile aqueous solution. $1 \mathrm{~mL}$ of this sample solution was detected by UFLC and repeated twice as above.

To determine the entrapment efficiency of $\beta$-elemene in elemene microemulsion, we compared the gel column chromatography and liquid surface method. As the column chromatography method had more errors, the liquid surface method was chosen. Because the free elemene as a volatile oil was insoluble in water and lighter in density than water, it would float on the surface of the aqueous phase. The internal solution of the microemulsion was thus taken to determine the entrapment efficiency. $2 \mathrm{~mL}$ of the elemene microemulsion stored at room temperature was moved into a tube as a test sample and set to stand for $2 \mathrm{~h}$. Then $0.1 \mathrm{~mL}$ of the internal solution of the test sample was taken out and diluted to $50 \mathrm{~mL}$ with the mobile phase to be measured for the $\beta$-elemene content as "a". Then $1.9 \mathrm{~mL}$ of the remaining solution of the test sample was diluted to $50 \mathrm{~mL}$ with the mobile phase, $1 \mathrm{~mL}$ of this diluted solution above was taken out and diluted again to $25 \mathrm{~mL}$ to be measured for the $\beta$-elemene content as "b". After repeated once as above, the average entrapment efficiency (EE) was calculated by the following formula:

$$
\begin{aligned}
\mathrm{C}_{\text {internal }} & =\mathrm{a} * 50 / 0.1, \mathrm{~W}_{\text {entrapment }}=\mathrm{C}_{\text {internal }} *\left(2 \mathrm{~mL}-\mathrm{V}_{\text {free }}\right) \\
& =500 * \mathrm{a} *\left(2 \mathrm{~mL}-\mathrm{V}_{\text {free }}\right), \\
\mathrm{W}_{\text {total }} & =\mathrm{a} * 50+\mathrm{b} * 25 * 50 . \\
\mathrm{EE} & =\mathrm{W}_{\text {entrapment }} / \mathrm{W}_{\text {total }} * 100 \%=10 * \mathrm{a} \\
& *\left(2-\mathrm{V}_{\text {free }}\right) /(\mathrm{a}+25 \mathrm{~b}) * 100 \% .
\end{aligned}
$$

$\mathrm{V}_{\text {free }}$ is the volume of elemene oil at liquid surface. As the oil phase was found to be insignificant in volume on the surface of the elemene microemulsion (at least $<0.05 \mathrm{~mL}$ ), EE should be more than $19.5 * \mathrm{a} /(\mathrm{a}+25 \mathrm{~b}) * 100 \%$.

\section{Oral relative bioavailability of elemene microemulsion in rats}

For the pharmacokinetics study, 90 Sprague Dawley rats weighing 140-200 g of both sexes were divided randomly into an elemene microemulsion group (45 rats) and an elemene emulsion group ( 45 rats) for oral administration by $100 \mathrm{mg} / \mathrm{kg}$ in elemene content. $3-5 \mathrm{~mL}$ of blood was drawn from femoral artery of rats at $0,0.5,1,1.5,2,2.5$, $3,4,6,8,10,12,14,18,24 \mathrm{~h}$ after administration (3 rats were used for each time point in each group). Then plasma was obtained after centrifuged for $10 \mathrm{~min}$ at $2000 \times \mathrm{g}$ and $4^{\circ} \mathrm{C}$, and stored at $4^{\circ} \mathrm{C}$. A mixture of $0.5 \mathrm{~mL}$ of plasma 
and $1 \mathrm{~mL}$ of acetonitrile was oscillated for $5 \mathrm{~min}$, set to stand for $5 \mathrm{~min}$ and then centrifuged for $30 \mathrm{~min}$ at $14000 \times \mathrm{g}$ and $25^{\circ} \mathrm{C}$. The supernatant was taken and filtrated with disposable needle filters $(0.22 \mu \mathrm{m})$. The filtrate was used for the content determination of $\beta$-elemene in plasma. Pharmacokinetic software DAS 2.0 was used for calculating pharmacokinetic parameters. Using UFLC detection, the standard curve of $\beta$-elemene concentration in plasma was linear in the range of $0.26-3.94 \mu \mathrm{g} \cdot \mathrm{mL}^{-1}$ with a regression equation of $\mathrm{Y}=2263.19 * \mathrm{C}-27.11(\mathrm{r}=0.9995)$.

\section{Statistic analysis}

Statistic analysis of the standard curve was carried out by OriginPro 7.5 software in fit linear moment. Statistic analysis of the pharmacokinetic study was carried out by DAS 2.0 software (Drug and Statistics software, Drug clinical research center, Shanghai University of Traditional Chinese Medicine, Shanghai, China) in statistical or compartmental moment. A value of $P<0.05$ was regarded as a significant difference.

\section{Results and discussion}

\section{Characterization of elemene} microemulsion

Many studies have shown that microemulsion may protect unstable drugs, increase the solubility of some insoluble drugs, improve bioavailability, control drug release, and reduce individual differences of drugs. ${ }^{5}$ The elemene microemulsion was characterized as $(57.7 \pm 2.8) \mathrm{nm}$ in size (Figure 2), $0.485 \pm 0.032$ in polydispersity index, $(3.2 \pm 0.4) \mathrm{mv}$ in zeta potential, $(5.19 \pm 0.08)$ in $\mathrm{pH}, 6 \mathrm{mpa} \cdot \mathrm{s}$ in viscosity, and $(31.8 \pm 0.3) \mathrm{mN} \cdot \mathrm{m}^{-1}$ in surface tension. Examination of the elemene microemulsion prepared in this work under crosspolarized light microscopy showed a dark field indicating an isotropic system. A 20-fold dilution of this microemulsion with water remained visually clear and dark under crosspolarized light microscope, indicating an excellent dispersing and isotropic stability. The elemene microemulsion as a clarified and isotropic system containing $1 \%$ elemene $(\mathrm{w} / \mathrm{v})$, $5 \%$ ethanol (v/v), $15 \%$ propylene glycol (v/v), $15 \%$ glycerol $(\mathrm{v} / \mathrm{v})$, and $5 \%$ polysorbate $80(\mathrm{w} / \mathrm{v})$ shows a formulation of weak acidity, low viscosity, and low surface tension that is suitable for oral administration. Generally speaking, 5\% ethanol $(\mathrm{v} / \mathrm{v}), 15 \%$ propylene glycol (v/v), 15\% glycerol (v/v), and $5 \%$ polysorbate $80(\mathrm{w} / \mathrm{v})$ could be tolerated by patients and pharmaceutical regulations. While mixing method was often used for the preparation of microemulsion, we found that it was easier to prepare an elemene microemulsion of greater clarity with ultrasonication in an ultrasonic bath, especially

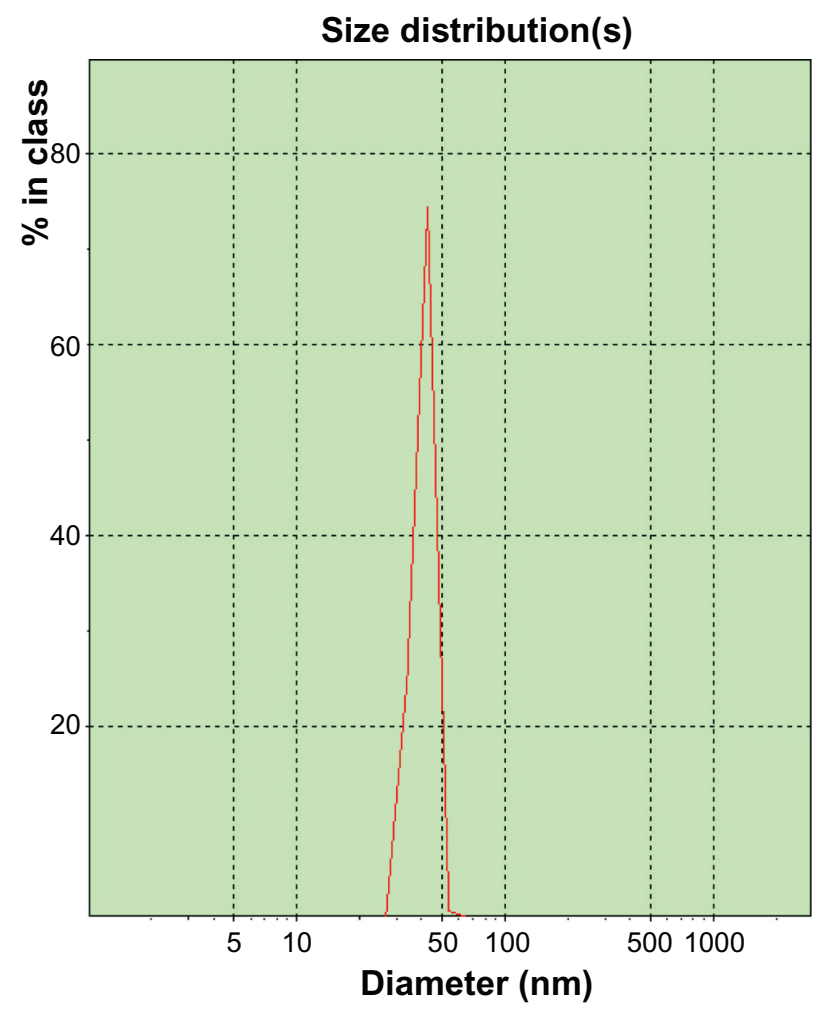

Figure 2 The particle size and size distribution of the elemene microemulsion.

when working around boundary conditions. In addition, the ultrasonication method can be easily adapted for production of elemene microemulsion at an industrial scale.

\section{Stability of elemene microemulsion}

When centrifuged at $25^{\circ} \mathrm{C}$ and $3000 \times \mathrm{g}$ and $50000 \times \mathrm{g}$ respectively for $30 \mathrm{~min}$, the phase separation was found in the case of the elemene emulsion, but not in the case of the elemene microemulsion indicating an excellent phase stability of the microemulsion.

\section{Content and entrapment efficiency of $\beta$-elemene in elemene microemulsion}

Currently gas chromatography or liquid chromatography technology is used for the content determination of elemene. During the samples preparation for gas chromatography, elemene is extracted from samples, dried and then dissolved again. ${ }^{12}$ Because elemene is a volatile oil and may be vaporized during the drying process, the quantitative result may not be accurate. This drying is not required in liquid chromatography for the analysis of drug content in elemene samples. In this work, UFLC was used to determine the drug concentration in the elemene preparations and rat plasma after oral administration. The content of $\beta$-elemene 
in the elemene microemulsion was determined to be $(8.273 \pm 0.018) \mathrm{mg} \cdot \mathrm{mL}^{-1}$.

Elemene is a volatile oil insoluble in water and lighter in density than water that the free elemene in an elemene microemulsion should float on the water surface. We failed to separate and determine the free elemene by dextran gel column chromatography, because insoluble elemene floated on the water or may be adsorbed to the gel particles. Therefore, the entrapment efficiency of elemene microemulsion was obtained by examining the drug concentration of the internal and total solutions using the liquid surface method. The average entrapment efficiency of $\beta$-elemene was determined to be $(99.81 \% \pm 0.24 \%)$.

\section{Plasma concentration-time curve and relative bioavailability of elemene microemulsion}

In the process of plasma samples treatment we found that the filter adsorption of drug on the $0.22 \mu \mathrm{m}$ filter was $3.6 \%$ and the extraction recovery of drug in plasma was $86.1 \%$. In the content determination of plasma samples, we found that the drug concentrations in plasma after oral administration of the elemene emulsion and microemulsion were very low. So, it was necessary to increase the ratio of plasma to acetonitrile in order to achieve a greater amount of elemene for detection. And $3-5 \mathrm{~mL}$ of blood was taken to obtain a sufficiently high drug concentration. After oral administration in rats, the fitting results of compartment model for the pharmacokinetic parameters of the elemene microemulsion were poor so that statistical moment method had to be used for calculating the pharmacokinetic parameters. The plasma concentration-time curves of the elemene microemulsion and emulsion are shown in Figure 3. The pharmacokinetic parameters were calculated by statistical moment as shown in the table insert. The result shows that the area under the concentration-time curves from $0 \mathrm{~h}$ to $24 \mathrm{~h}\left(\mathrm{AUC}_{0 \rightarrow 24 \mathrm{~h}}\right)$ of the elemene emulsion and microemulsion was $1.896 \mathrm{mg} \cdot \mathrm{h} \cdot \mathrm{L}^{-1}$ and $3.092 \mathrm{mg} \cdot \mathrm{h} \cdot \mathrm{L}^{-1}$ respectively, yielding a relative bioavailability of $163.1 \%$. The $\mathrm{C}_{\max }$ of the elemene microemulsion was $1.820 \mathrm{mg} . \mathrm{L}^{-1}$ and 1.3 times of the elemene emulsion. The MRT (mean residence time) of the elemene microemulsion was $1.668 \mathrm{~h}$, greater than that of the elemene emulsion. But the standard deviations of the average drug concentrations were relatively large indicating large individual differences for oral absorption.

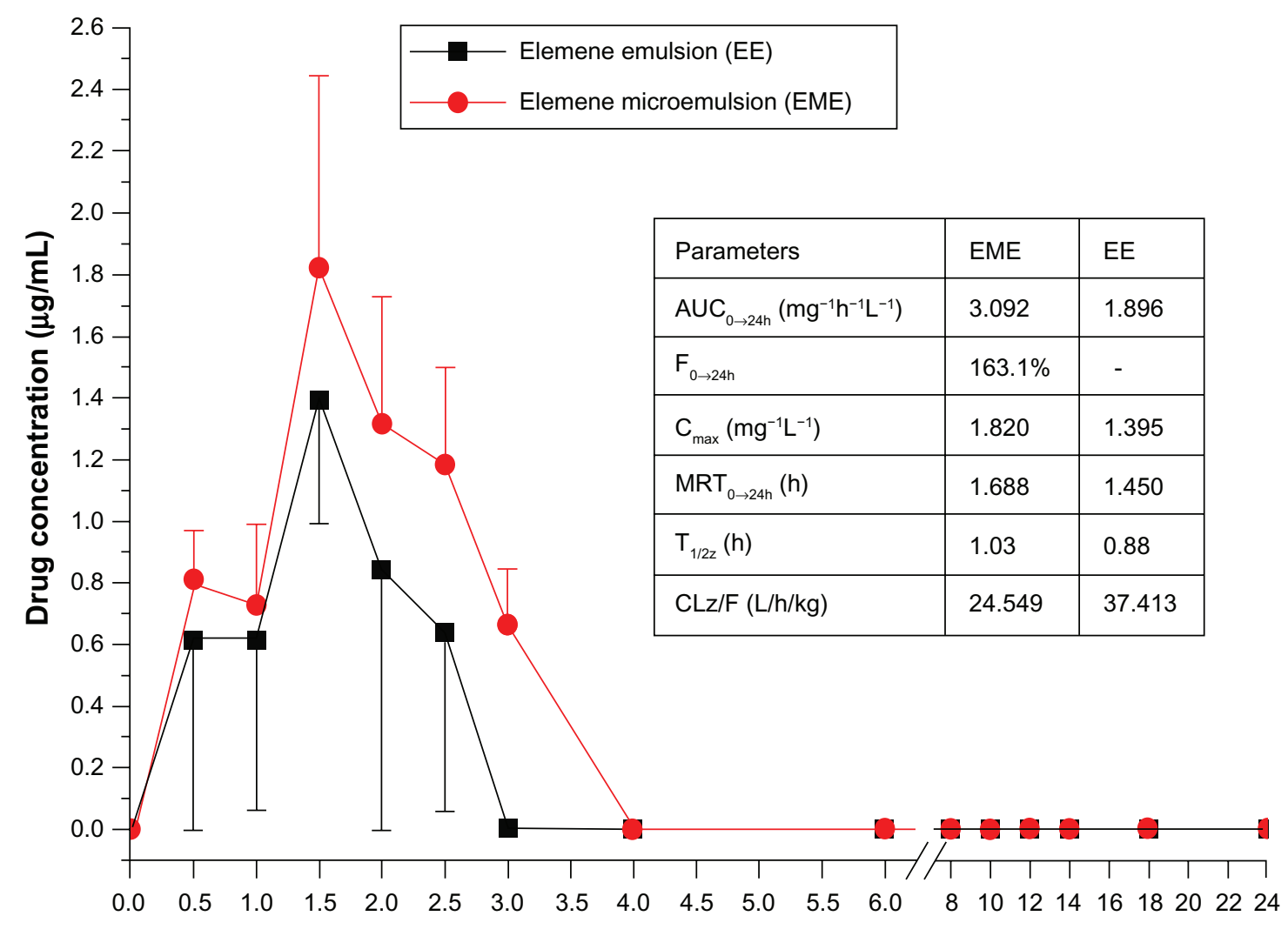

Time (h)

Figure 3 The plasma concentration-time curves of the elemene microemulsion and emulsion. 
In other studies docetaxel $\mathrm{o} / \mathrm{w}$ microemulsion is reported to have a relative bioavailability of $519.2 \%$ over taxotere,${ }^{6}$ while the relative bioavailabity of cyclosporine microemulsion was enhanced with a $16 \%$ to $31 \%$ increase in AUC and a $32 \%$ to $42 \%$ increase in $\mathrm{C}_{\max }{ }^{13}$ Our findings with elemene microemulsion in this work were in agreement with the literature reports. Although the enhanced extent of bioavailability of the elemene microemulsion is a little less than desired, the surfactant content used is a little lower. ${ }^{6,13,14}$ These results encourage further development of elemene microemulsions as an oral drug delivery system.

\section{Conclusion}

This study shows that the elemene o/w microemulsion with good clarity, excellent stability, high entrapment efficiency, and improved bioavailability can be prepared successfully using elemene as the oil phase and drug, polysorbate 80 as the surfactant along with ethanol, propylene glycol, glycerol as the cosurfactants, and ultrasonication as the dispersing force.

\section{Acknowledgment}

The work was supported by grants from Foundation of Zhejiang Science and Technology Department (2009C33005), National Natural Science Foundation of China (20906016), Technology Research and Development Program of Hangzhou (20090331N03), and "Biomedical Engineering" Top Priority Open Funds of ZheJiang (SWYX0919).

\section{Disclosure}

The authors report no conflicts of interest. The authors are solely responsible for the content and writing of the paper.

\section{References}

1. Sun MG, Li SB, Zhou L, et al. Chemical constituents of active pharmaceutical ingredients of elemene. J Shenyang Pharm Univ. 2009;26(8):620-622.

2. Chen X, Wei FX, Qu YH. Development in the study of elemene - the active principle of Rhizoma Curcumae. Hebei Chemical Engineering Industry. 2003;(6):9-13.

3. Yao SJ, Liu BY, Lv LY. Studies of reducing poison and increasing effect of elemene emulsions on immune function of tumor-bearing mice after chemiotherapy. Chin Arch Trad Chin Med. 2006;24(3):456-457.

4. Xu XX, Zhuang ZM. Recent advance of pharmacological effects and clinical application of elemene emulsion. Strait Pharm. 2001;13(2): 7-8.

5. Yang H, Yi H. Advance on pharmaceutical microemulsion in China. Chin J New Drugs. 2006;15(10):764-769.

6. Yin YM, Cui FD, Mu CF, et al. Docetaxel microemulsion for enhanced oral bioavailability: Preparation and in vitro and in vivo evaluation. J Control Release. 2009;140(2):86-94.

7. Zhou Y, Liu W. Advance on pharmaceutical application of microemulsion and self-microemulsion drug delivery system. J Northwest Pharm. 2008;23(1):56-58.

8. Yang R, Su LQ, Huang X. Study advance and application of microemulsion in pharmaceutics. Chin J Hospital Pharm. 2007;27(8):1141-1144.

9. The Ministry of Science and Technology of the People's Republic of China. Beijing: Guideline of laboratory animal care [online]. Accessed Jul 11, 2010. Available from: http://www.most.gov.cn/fggw/zfwj/ zfwj2006/200609/t20060930_54389.htm

10. Hathout RM, Woodman TJ, Mansour S, et al. Microemulsion formulations for the transdermal delivery of testosterone. Eur J Pharm Sci. 2010;40(3):188-196.

11. Zeng ZW, Zhou GL, Zhan XR, et al. Determination of content and entrapment efficiency of elemene liposomes with UPLC method. Chin J Pharm Anal. 2010;30(3):508-510.

12. Zhang XY, Deng YJ, Zhao CJ, et al. Study on pharmacokinetics of PVP coated $\beta$-elemene liposome in rats. J Chin Med Mater. 2006; 9(2):157-160

13. Barone G, Chang CT, Choc MG Jr, et al. The pharmacokinetics of a microemulsion formulation of cyclosporine in primary renal allograft recipients. The Neoral Study Group. Transplantation. 1996;61(6): 875-880.

14. Bali V, Ali M, Ali J. Study of surfactant combinations and development of a novel nanoemulsion for minimising variations in bioavailability of ezetimibe. Colloids Surf B: Biointerfaces. 2010;76(2):410-420.
International Journal of Nanomedicine

\section{Publish your work in this journal}

The International Journal of Nanomedicine is an international, peerreviewed journal focusing on the application of nanotechnology in diagnostics, therapeutics, and drug delivery systems throughout the biomedical field. This journal is indexed on PubMed Central, MedLine, CAS, SciSearch ${ }^{\circledR}$, Current Contents ${ }^{\circledR} /$ Clinical Medicine,

\section{Dovepress}

Journal Citation Reports/Science Edition, EMBase, Scopus and the Elsevier Bibliographic databases. The manuscript management system is completely online and includes a very quick and fair peer-review system, which is all easy to use. Visit http://www.dovepress.com/ testimonials.php to read real quotes from published authors. 